\title{
Mathematical Evaluation of Context Transfer and Multicast Fast Reroute in Multicast Enabled Network Mobility Management
}

\author{
Azana Hafizah Mohd Aman ${ }^{1}$, Aisha-Hassan A. Hashim² and Huda Adibah Mohd \\ Ramli \\ Kulliyyah of Engineering, International Islamic University Malaysia, Jalan \\ Gombak 53100, Kuala Lumpur, Malaysia \\ 'azana05@yahoo.com,2aisha@iium.edu.my
}

\begin{abstract}
Internet applications such as web based monitoring; live internet video, online video, video conference, webcam viewing and internet video to TV are highly used in today's IP communication. The trends of these applications are, that they are played on mobile devices and distributed to many end users. Multicast communication over IP contributes to the end users applications distribution. It has been discovered by a CISCO research that mobile multicast traffic will soon reach zetabyte in 2019. The aim of this paper is to introduce new method that enable multicast in network mobility management. The new method is using context transfer and multicast fast reroute technique. The proposed method is quantitatively evaluated in terms of packet loss and service recovery time
\end{abstract}

Keywords: Context Transfer, Fast Reroute, Mobile Multicast, Network Mobility

\section{Introduction}

Network performance issues due to extreme traffic demand from mobile users have attracted many researchers. Among of the network performance issues are packet loss, service recovery time, router discovery delay, signaling cost and many more. Network service providers claim for latest high multicast technology that can provide acceptable service recovery time and low packet loss. As for mobile multicast communication, it is compulsory to reconstructs Multicast Listener Discovery (MLD) [1] states in a mobile multicast communication. Approach of transferring this state affects service recovery time for multicast communication.

This paper is organized as follows; Section 2 presents related works and current issues. Section 3 highlights integration consideration of multicast PMIPv6, context transfer and multicast fast reroute. Section 4 describes design implementation of the proposed integration. Section 5 clarifies the performance result and discussion of the selected parameters. Finally, the conclusion of the paper is presented in Section 6.

\section{Related Works and Current Issues}

The Proxy Mobile IPv6 (PMIPv6) [2] is well known as a unicast network based mobility management, is an extension of Mobile IPv6 (MIPv6) [3]. It enables IP mobility for a host without the involvement of any mobility-related signaling. Two new network entities named as Local Mobility Anchor (LMAs) and Mobile Access Gateways (MAGs) are in charge of the mobile node (MN) IP mobility signaling. Basically as a unicast communication, participation in multicast communication is tedious. The reason is due to the fact that MAGs discard group or multicast packets. Presently there are few multicast enabled PMIPv6 proposals. These proposals are direct routing, route optimization, context transfer, global mobility and load balancing. 
Route optimization proposals offer optimal delivery path for multicast data in PMIPv6. Hence deliver minimum service disruption time as there is no extra binding update delay nor multicast tree join delay. Most proposals decreases the packet lost rate by modifying the buffering and forwarding multicast data process during mobile node handover. Context transfer proposals add an extension of the Context Transfer Protocol (CXTP) in PMIPv6 to provide smooth handover. These proposals offer multicast session continuousness and avoids further session disruption and packet loss.

Global mobility proposals offer inter domain (global) multicast mobility between PMIPv6 domains. These proposals support the global mobility by adding extra signaling messages between LMAs. Also, it achieves low latency because it performs fast binding and group joining procedure. Load balancing proposals tackle multicast traffic bottleneck issue at the LMAs. Load balancing among LMAs is helps to solve bottleneck issue. It distributes the load equally among the LMAs hence minimizing the multicast service disruption.

One of the proposals is to deploy Multicast Listener Discovery (MLD) [1] multicast routing functions at Local Mobility Anchors (LMAs) and proxy functions at Mobile Access Gateways (MAGs) [4]. This basic deployment [4] shadows the standard general PMIPv6 [2] traffic type. Another proposal introduces method for multicast route implementation straight at the access gateways [5]. Others selected selective route enhancement as to simplify multicast PMIPv6 routing [5-7]. These proposals basically follow the typical type of servicing multicast communication in corresponding to PMIPv6 unicast route [8].

Proposal [9] investigated and implemented an extension towards the existing MIPv6 to enable PMIPv6 model, configured 6 nodes with 1 mobile node. Making used of the UDP (User Datagram Protocol) and TCP (Transmission Control Protocol) traffics. Performance metrics considered are average handover delay, average packet delivery ratio and throughput. Through simulation experiments value collected are number of packets received, number of packets sent and number of bytes receive. It proved that the suggested scheme satisfied the movement mobility of PMIPv6 however it does not consider distributed communication or multicast communication.

Proposal [10] presented PMIPv6 with Multi-Protocol Label Switching (MPLS). It used xMIPv6 as a base, and modified it to support the MPLS/PMIPv6 protocol. Performance metrics considered are handover delay, handover overhead and end-to-end delay. Simulations are varied by number hop between MAG and LMA. [11] enhanced flow mobility support using multiple host interface and the mobility practice in the suggested scheme. Both proposals [10] and [11] focused on multi-protocol and interfaced, therefore lack in details for mobility multicast communication.

\section{Integration Consideration}

This paper considers two important methods, which are predictive type of Context Transfer (CT) [12-13] and Multicast only Fast Reroute (MFR) [14]. These two considerations are very important since it can improve the performance in proxy mobile multicast environment especially in a very dense mobile multicast traffic. It is a new improvement for the present PMIPv6, by integrating PMIPv6 with MFR and CT. The aim of this paper is to enhance the service recovery performance and to provide low packet loss in PMIPv6 multicast communication.

Multicast Fast Reroute [14] defines a tool for reducing packet loss in a network when link or node disasters occur. MFR defines two paths, a main path and a backup path. Both paths join the tree concurrently and both obtain multicast traffic. But only the packets from the main are accepted and forwarded, as the packets from the backup are rejected. When the primary failure is detected, the restoration takes place by swapping the path. It 
is a local swapping, therefore it is fast and greatly refining convergence times when link or node disasters occur. Figure 2 shows the standard network design path of MFR.

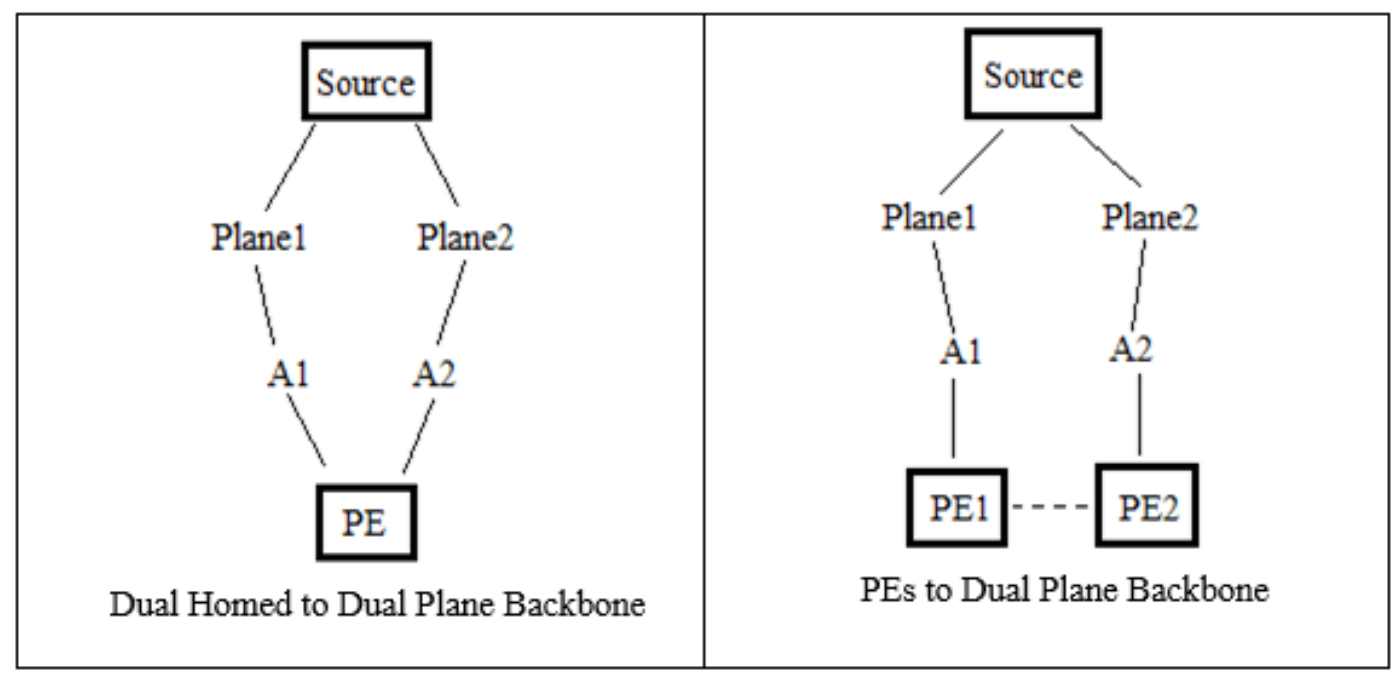

Figure 2. Network Design of MFR

Figure 2 shows that the topology has two planes, a primary plane and a secondary plane that are fully disjoint from each other. Multicast traffics for densely watched communication flow on both the planes. PE is a router that obtains two copies of the traffic.

CT [12-13] is used to transfer context of services. Mobile nodes (MN) with context transfers highly tolerate applications running on it to function under low disruption. Context Transfers Protocol provides very high support in terms of optimized handover performance. It leads to optimized mobile node performance in mobile multicast environment. CT minimizes the re-establishment of services from scratch, reduces latency and minimizes packet losses. The Context Transfer protocol normally works between a source node and a target node. A source is an MN's old access router and the destination is an MN's new access router. When this occurred, all nodes communicate to each other accordingly. The MN handover function flow in a predictive handovers environment for the proposed integration is shown in Figure 3.

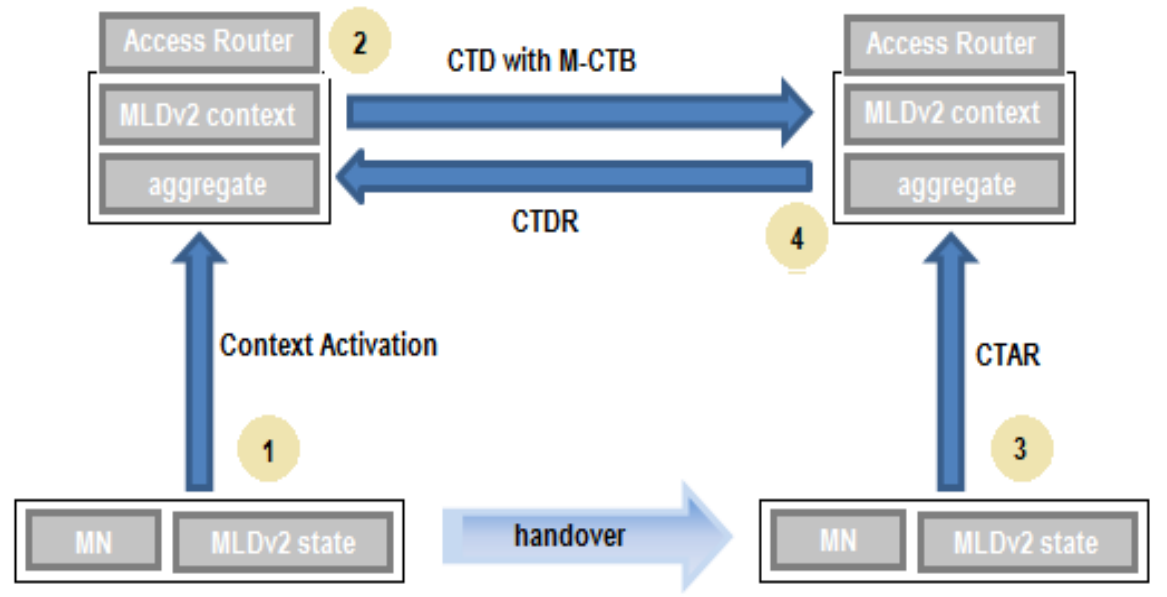

Figure 3. Basic Context Transfer 


\section{Design Implementation}

As Figure 4, Figure 5 and Figure 6 shows the network architecture, context transfer process and signaling call flow for the integration of CT and MFR with PMIPv6 respectively. The MAGs are the provider edge. Figure 5 shows the changing function of main and backup PE as the mobile node connection changes. When the $\mathrm{MN}$ is linked to old MAG (pMAG), the LMA sends the multicast data to both PE1 and PE2. The pMAG rejects one of the multicast traffic and sends the other multicast traffic to the mobile node.

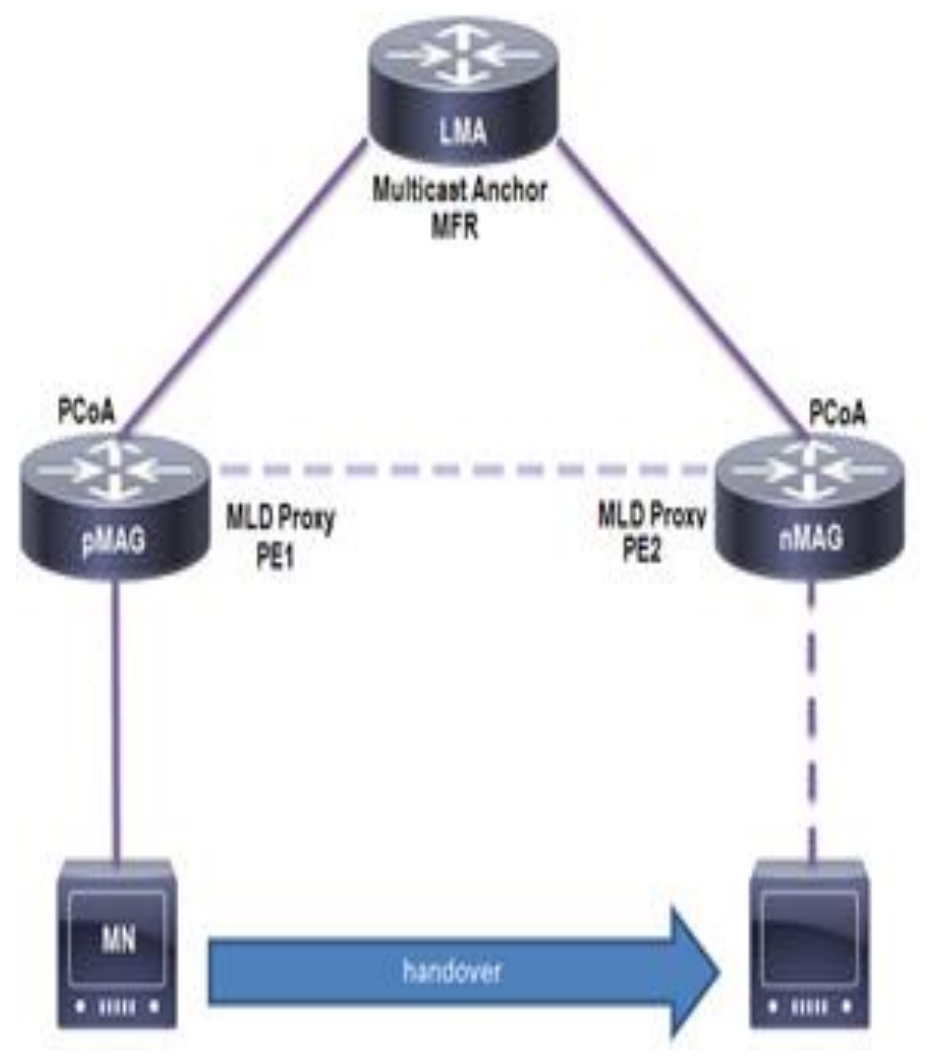

Figure 4. Network Model of CTMFR 


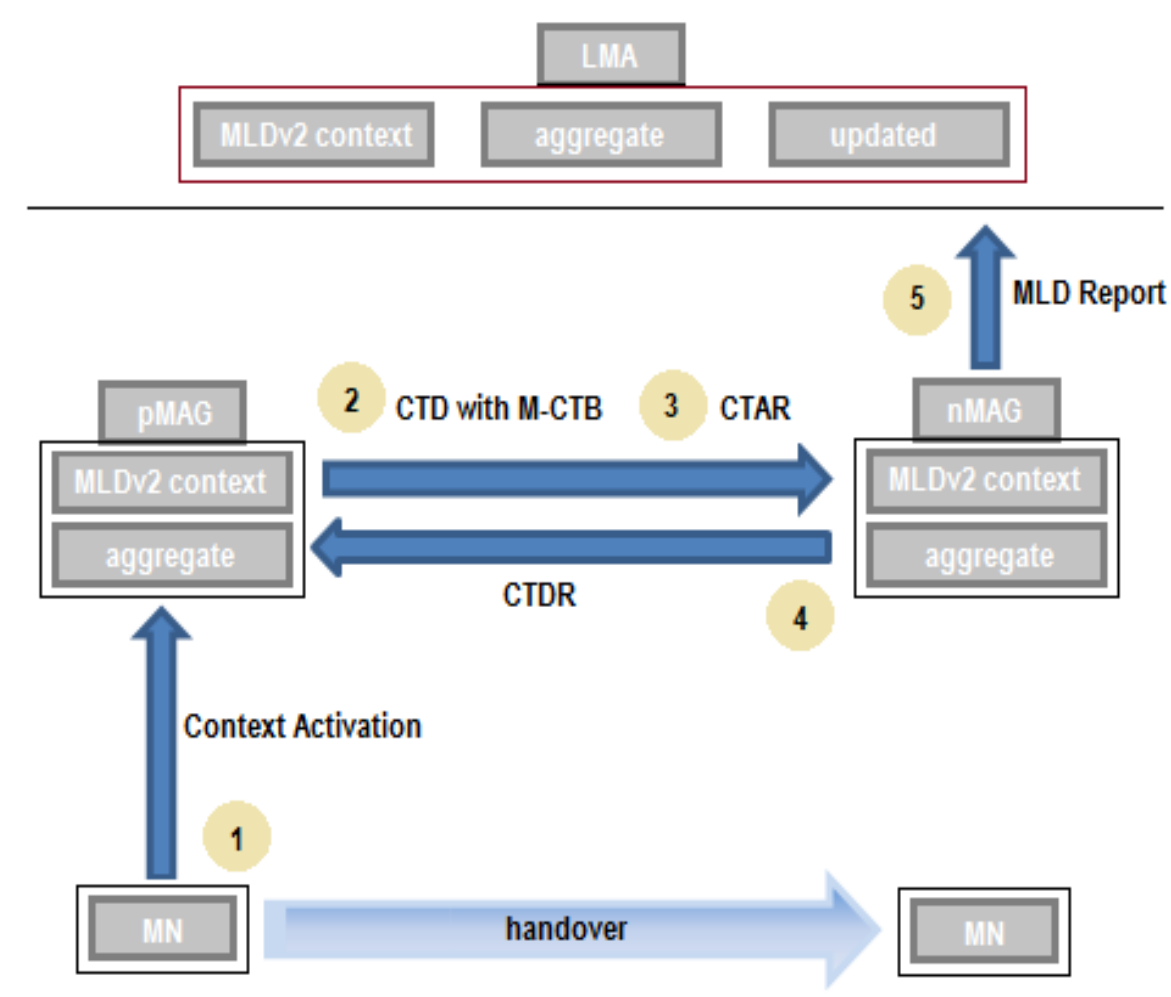

Figure 5. Predictive Multicast Context Transfer Operation PMIPv6

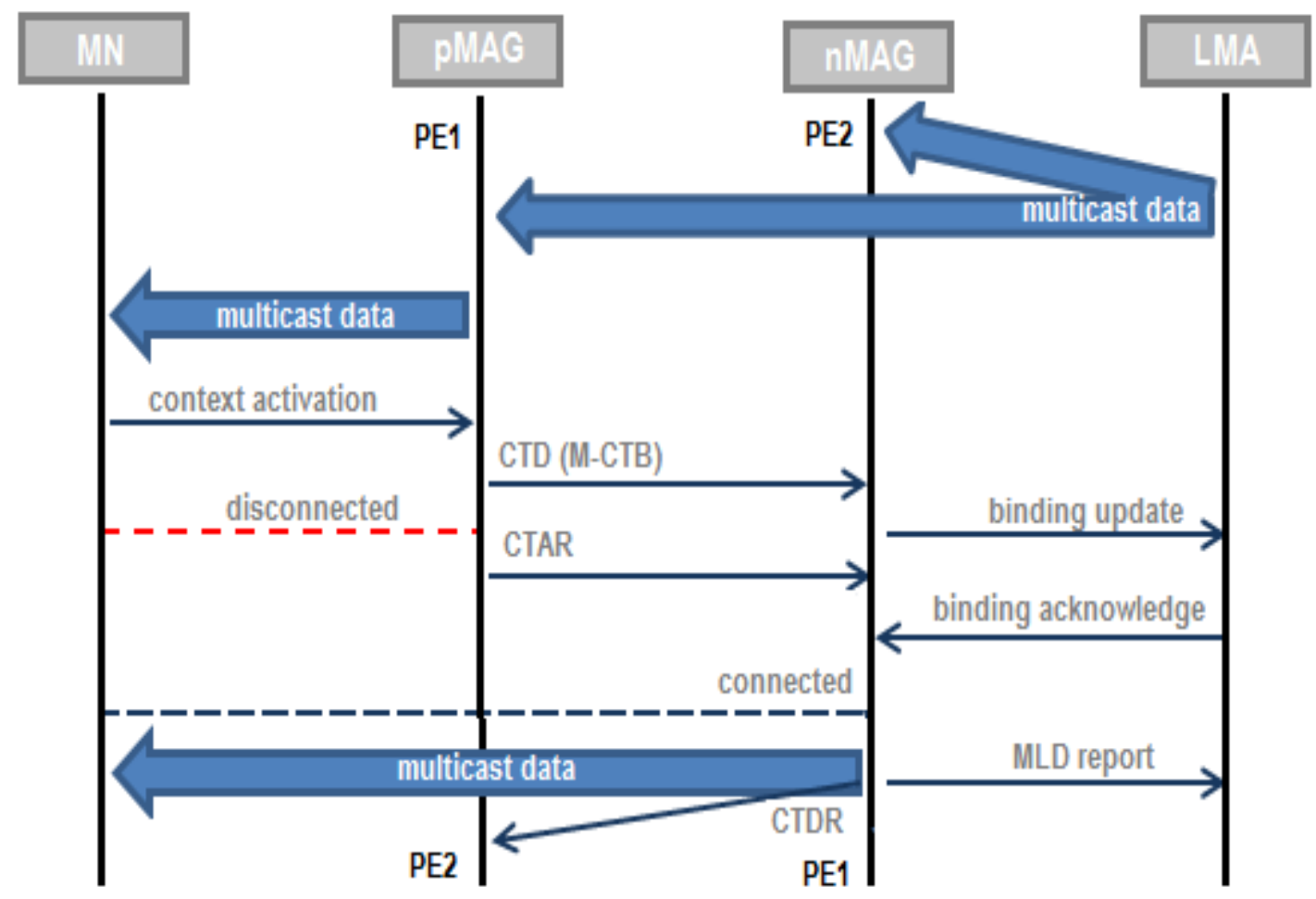

Figure 6. Signaling Call Flow for the Integration 


\section{Analysis and Discussion}

The integration is analyze for its packet loss cost and service recovery time. The values and parameters are subjected to [15-16].

The service recovery time is described as the time needed for the service to resume to normal state. The total service recovery time is denoted as $\mu_{\mathrm{t}}$. Table 1 describes the parameters used.

Table 1. Parameters for Total Service Recovery Time

\begin{tabular}{|c|l|}
\hline Parameter & \multicolumn{1}{c|}{ Description } \\
\hline$\delta_{C T A R}$ & $\begin{array}{l}\text { time taken to send the Context Transfer Active Request } \\
\text { message from pMAG to nMAG }\end{array}$ \\
\hline$\delta_{B A}$ & $\begin{array}{l}\text { time taken to send the Binding Acknowledge message } \\
\text { from LMA to nMAG }\end{array}$ \\
\hline$\delta_{M D 1}$ & $\begin{array}{l}\text { time taken to send the first Multicast Data message from } \\
\text { LMA to nMAG }\end{array}$ \\
\hline
\end{tabular}

The total service recovery time, $\mu_{\mathrm{t}}$ is elaborated in equation 1 :

$$
\mu_{\mathrm{t}}=\delta_{C T A R}+\delta_{B A}+\delta_{M D} \text {. }
$$

The value for $\delta_{C T A R}$ is $10 \mathrm{~ms}$, the value for $\delta_{B A}$ is $10 \mathrm{~ms}$ and the value for $\delta_{M D I}$ is $10 \mathrm{~ms}$. The packet loss cost is calculated from the packet arrival rate, and service recovery time. Table 2 describes the parameters used. The packet loss cost is noted as $\alpha$.

Table 2. Parameters Packet Loss Cost

\begin{tabular}{|c|l|}
\hline Parameter & \multicolumn{1}{c|}{ Description } \\
\hline$\beta$ & packet arrival rate \\
\hline$\delta_{C T A R}$ & $\begin{array}{l}\text { time taken to send the Context Transfer Active Request } \\
\text { message from pMAG to nMAG }\end{array}$ \\
\hline$\delta_{B A}$ & $\begin{array}{l}\text { time taken to send the Binding Acknowledge message } \\
\text { from LMA to nMAG }\end{array}$ \\
\hline$\delta_{M D 1}$ & $\begin{array}{l}\text { time taken to send the first Multicast Data message } \\
\text { from LMA to nMAG }\end{array}$ \\
\hline
\end{tabular}

However in case of MPMIPv6 with RCMFR, $\alpha$ is described in equation 2;

$$
\alpha=\beta\left(\delta_{C T A R}+\delta_{B A}+\delta_{M D}\right)
$$

Figure 7 shows the service recovery versus router discovery delay. 


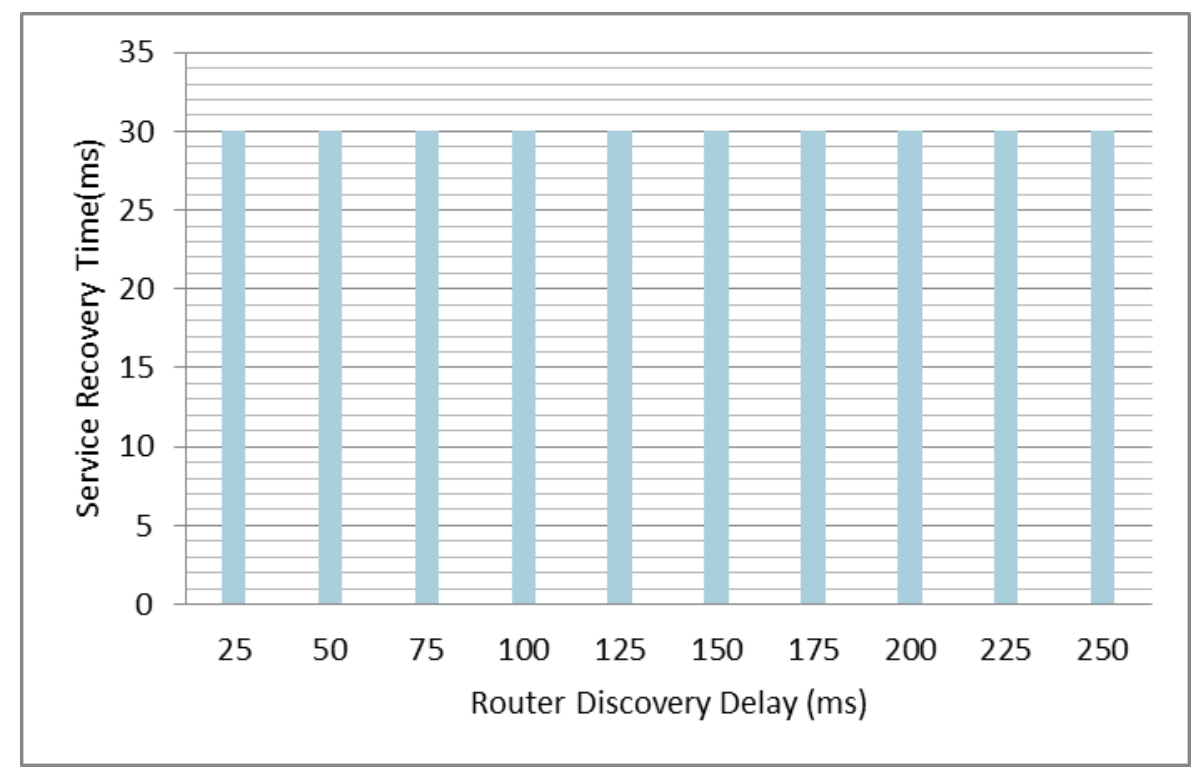

Figure 7. Service Recovery Time versus Router Discovery Delay

As shown in Figure 7, the service recovery time for the integration method remain constant at the value of $30 \mathrm{~ms}$, regardless of the increasing value of the router discovery delay. This is because the integration clearly eliminates the router discovery dependency therefore unrelatedly with the discovery time increment the service recovery time remain the same. As Figure 8 illustrates the packet loss cost versus packet arrival rate. As the packet arrival rate increases the packet loss cost increases, at an increment of 0.5 packet for each 10packet/s.

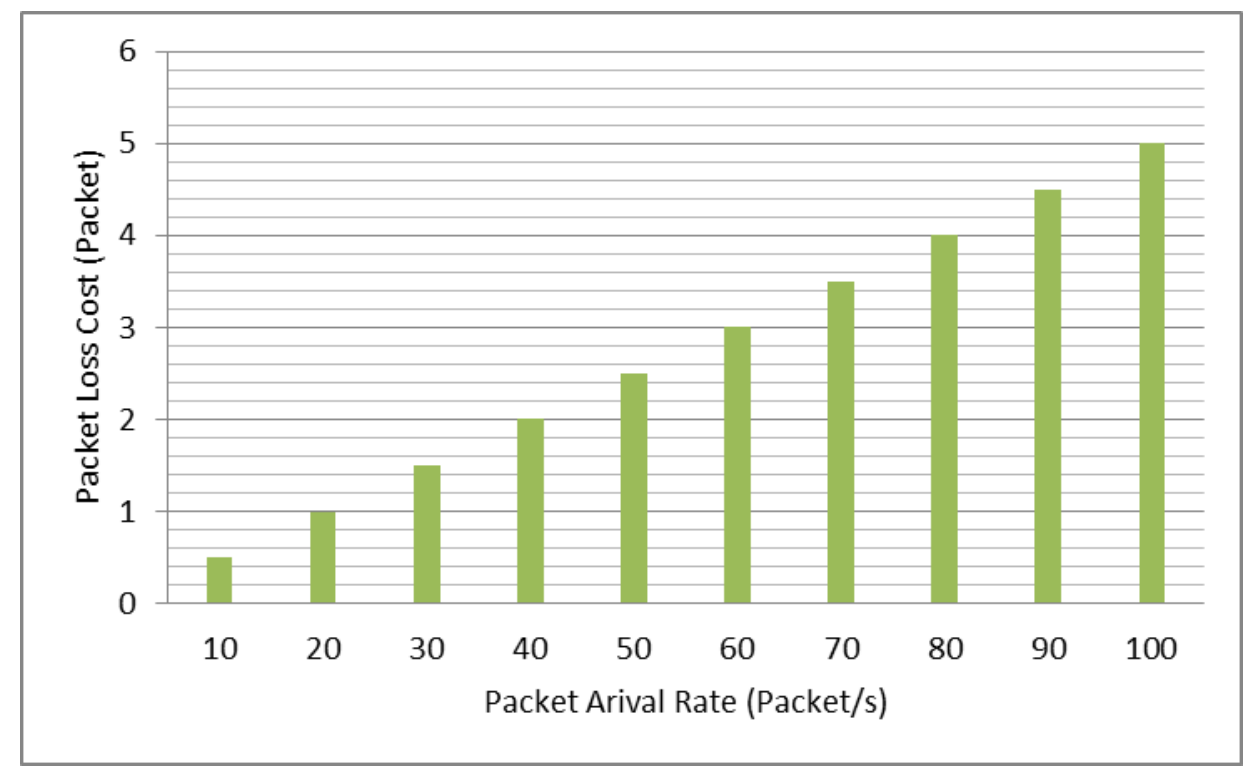

Figure 8. Packet Loss Cost versus Packet Arrival Rate

In Figure 9, the percentage of packet loss versus packet arrival rate is displayed. As shown the value of maximum loss is only $5 \%$ which is still below acceptable loss percentage. The percentage of packet loss decreases as the packet arrival rate increases. The decrease rate is $0.5 \%$ for each 10 packet/s. 


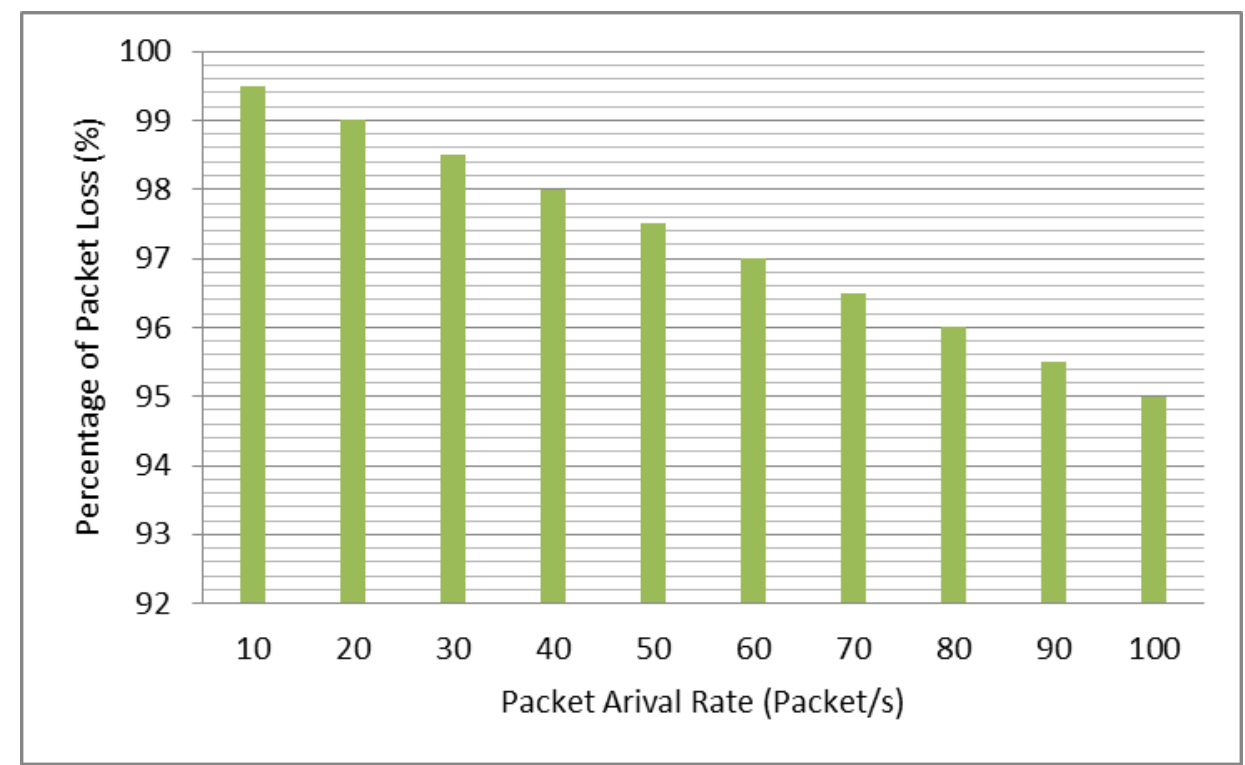

Figure 9. Percentage of Packet Loss Cost versus Packet Arrival Rate

Figure 10 shows packet loss cost versus link delay. As the link delay increases the packet loss cost increases. The increase rate for packet loss cost is 0.1 packet for each 50s.

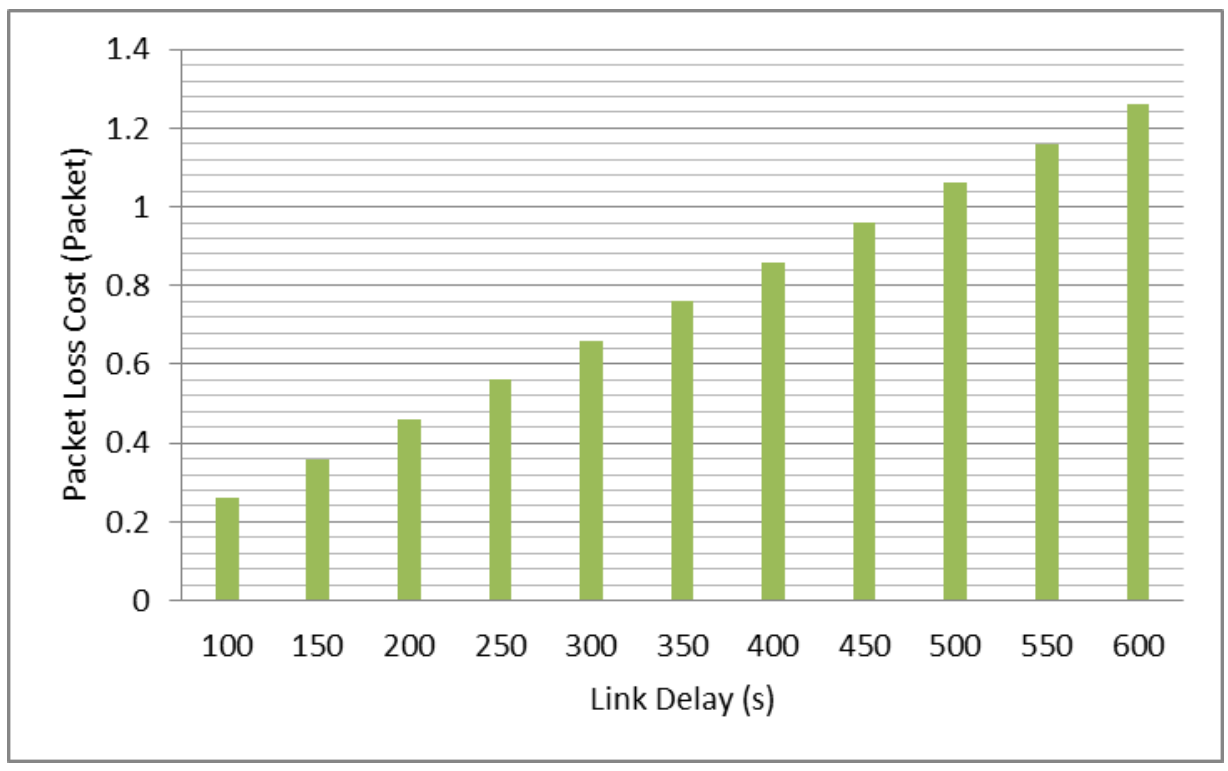

Figure 10. Packet Loss Cost versus Link Delay 


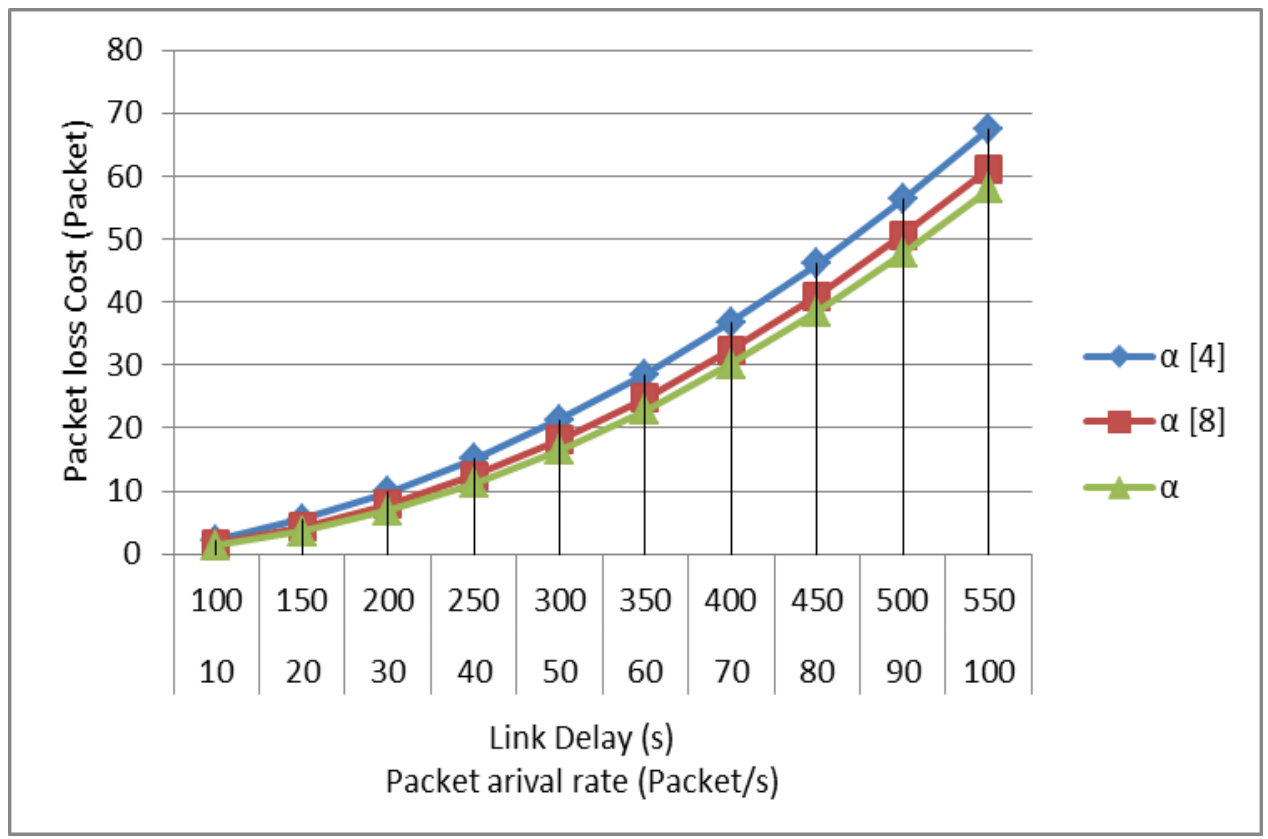

Figure 11. Packet Loss Cost versus Packet Arrival Rate and Handover Latency

Overall from Figure 11, the packet loss cost for the integration method is the lowest compared to the work in $[4,8]$. In Figure 8, as the packet arrival rate increases, the packet loss cost increases but in small and consistent manner. Same situation with Figure 10, as the link delay increases, the packet loss cost increases but in small and consistent manner. As for work in $[4,8]$ bigger packets drop in case of traffic interruption. But since the MFR is implemented, when there is network failure the swapping of primary and secondary path will occur. This integration enables two-plane implementation towards multicast enabled PMIPv6. This integration removes high service recovery time and high packet loss cost. Therefore the probability of losing packet is very minimal. It is obvious that the integration offers considerable and acceptable packet loss cost. Hence by using this method, multicast mobility achieves the fastest service recovery time, low packet loss cost and low percentage of packet loss.

\section{Conclusion}

The standard PMIPv6 mainly support unicast routing only and with no consideration for multicast routing. Therefore PMIPv6 multicast is still an ongoing concept to study. Recent proposals are up to enabling multicast support but there has not been a multicast PMIPv6 standardization protocol presented. This has motivated the integration of CT and MFR methods to provide low service recovery time and low packet loss for mobile multicast communication. As to validate these mathematical results, experimental approach is considered as future work. 


\section{References}

[1] R Vida, L Costa, "Multicast Listener Discovery Version 2 (MLDv2) for IPv6”, RFC 3810 (2004).

[2] Gundavelli S, Ed Leung, K Devarapalli, V Chowdhury K and B Patil, "Proxy Mobile IPv6 (PMIPv6)", RFC 5213 (2008) .

[3] Johnson, D., Perkins, C., and J. Arkko, "Mobility Support in IPv6", RFC 6275 (2011) July.

[4] T Schmidt, M Waehlisch and S Krishnan, "Base Deployment for Multicast Listener Support in Proxy Mobile IPv6 (PMIPv6) Domains", RFC 6224, (2011).

[5] JC. Zuniga, LM. Contreras, CJ. Bernardos, S. Jeon, Y. Kim , "Multicast Mobility Routing Optimizations for Proxy Mobile IPv6", RFC 7028 (2013).

[6] J Liu, W Luo and W Yan, "Routes Optimization for PMIPv6 Multicast", Internet-Draft (2012).

[7] Tsunehiko Chiba, Hidetoshi Yokota, Ashutosh Dutta, Dana Chee, Henning Schulzinne, "Route Optimization for Proxy Mobile IPv6 in IML Network", 2nd International Conference on Signal Processing and Communication Systems (ICSPCS 2008), (2008).

[8] QUALNET http://web.scalable-networks.com/content/qualnet

[9] Farouk Abdul Jalin and Raed Alsaqour, "A Simulation Study of Proxy Mobile IPV6 (PMIPV6) Protocol", J. of Engineering and Applied Sciences, vol. 11(7), (2016).

[10] LM. Contreras, CJ. Bernardos, I. Soto, "Proxy Mobile IPv6 (PMIPv6) Multicast Handover Optimization by the Subscription Information Acquisition through the LMA (SIAL) “, RFC 7161 (2014).

[11] Farouk Abdul Jalin and Raed Alsaqour, "A Simulation Study of Proxy Mobile IPV6 (PMIPV6) Protocol", J. of Engineering and Applied Sciences, vol. 11(7), (2016)

[12] Hatsadin Payappanon, Thossaporn Kamolphiwong, Kevin Robert Elz, "Simulation and Evaluation of MPLS based PMIPv6 Network", International J. of Advances in Computer Science and Technology, Vol.2 (8), (2013) Pp: 07-11,

[13] G.S. Tomar \& Shekhar Verma "Analysis of handoff Initiation using different path loss models in mobile communication system" $3^{\text {rd }}$ IEEE International conference on wireless and optical communication networks "WOCN2006", pp-5, 11-13 Apr 2006

[14] Hyon-Young Choi, Sung-Gi Min, Youn-Hee Han and Rajeev Koodli, "Design and Simulation of a Flow Mobility Scheme Based on Proxy Mobile IPv6”, J Inf Process Syst, Vol.8 (4), (2012).

[15] K.Mayuri and K.S.Ranjith, "A Novel Secure Handover Mechanism In Pmipv6 Networks", International J. of Information Technology Convergence and Services, Vol.4 (4), (2014).

[16] A. Karan, C. Filsfils, IJ. Wijnands, and B. Decraene, "Multicast Only Fast Reroute (MoFRR)" ,RFC 7431, (2015) August.

[17] Moneeb Gohar, Sang Il Choi, Seok Joo Koh, "Fast handover using multicast handover agents in PMIPv6-based wireless network" The 25th International Conference on Information Networking (ICOIN 2011), (2011) January.

[18] S. Verma, GS Tomar, "Call Admission Control and Handoff Techniques for 3-G and Beyond Mobile Network", Asia-pacific Journal of Multimedia Services Convergence with Art, Humanities and Sociology, Vol.1, No.1, pp. 31-42, 2011.

[19] Adnan J.Jabir, S. Shamala and Z.Zuriati, "A new strategy for signalling overhead reduction in the proxy mobile IPv6 Protocol”, Am. J. of Applied Sci., vol. 9, issue 4, (2012), pp. 535-541. 